\title{
Pólipo endometrial, una causa infrecuente de sangrado genital anormal en la adolescencia
}

\author{
Constanza Ralph $T_{i}^{1}$, Claudia Zajer A. $^{1}$, Valentina De Petris V. ${ }^{a}$, Roger Gejman E. ${ }^{2}$, \\ Mauricio Cuello $F^{1}$ \\ ${ }^{1}$ División de Obstetricia y Ginecología, ${ }^{2}$ Departamento de Anatomía Patológica. Escuela de Medicina, Pontificia Universi- \\ dad Católica de Chile.
}

a Alumna. Escuela de Medicina, Pontificia Universidad Católica de Chile.

\section{RESUMEN}

Antecedentes: El sangrado genital anormal es una causa frecuente de consulta en la adolescencia. En este período, la principal causa de sangrado es la metrorragia asociada a ciclos anovulatorios producto de la inmadurez del eje hipotálamo-hipofisario-gonadal. Dentro de las causas infrecuentes de sangrado genital anormal en ese período está el pólipo endometrial. Caso clínico: Presentamos el caso de una niña de 13 años cuya causa de sangrado correspondió a un pólipo endometrial, sospechado por ultrasonografía, resecado mediante histeroscopia y confirmado mediante estudio histológico. Discusión: Pese a su baja incidencia, los pólipos endometriales deben ser considerados como parte del diagnóstico diferencial en adolescentes que consultan por trastorno menstrual, particularmente en aquellos casos sin respuesta a la terapia hormonal y donde la ultrasonografía muestra engrosamiento endometrial.

\section{PALABRAS CLAVES: Pólipo endometrial, adolescencia, metrorragia}

\section{SUMMARY}

Background: Abnormal genital bleeding is a common cause of medical consultation in patients during adolescence. In this period, the main cause of genital bleeding is metrorrhagia in relation to anovulatory cycles due to immaturity of hypothalamus- pituitary-gonadal axis. Among the uncommon causes of bleeding at this age is the endometrial polyp. Case report: We report a 13 year old girl with abnormal uterine bleeding due to endometrial polyp, suspected during a pelvic ultrasound, removed by hysteroscopy, and confirmed by histological analysis. Discussion: Despite its low incidence, endometrial polyps should be included in the differential diagnosis of adolescents presenting menstrual disorders, particularly in those with no response to hormonal therapy and endometrial thickness in ultrasound.

\section{KEY WORDS: Endometrial polyp, adolescence, metrorrhagia}




\section{INTRODUCCIÓN}

El sangrado genital anormal, debido a trastornos del ritmo menstrual, representa el $50 \%$ de las consultas ginecológicas durante la adolescencia $(1,2,3)$. La mayoría de los casos son atribuibles a metrorragia originada en ciclos menstruales anovulatorios producto de la inmadurez del eje hipotálamo-hipofisiario-gonadal. Otras causas comunes de sangrado son el trauma genital, la presencia de un cuerpo extraño en la vagina, las infecciones genitales y las malformaciones uterinas.

Los pólipos endometriales son poco frecuentes en mujeres menores de 20 años (4), y una causa inhabitual de sangrado genital anormal durante la adolescencia. Como entidad, los pólipos endometriales corresponden a una proliferación circunscrita del endometrio, con un eje conjuntivo vascularizado, los cuales pueden presentarse en forma aislada o en forma múltiple al interior de la cavidad uterina. Su incidencia aumenta progresivamente con la edad, alcanzando su máxima incidencia en la quinta década de la vida, para posteriormente disminuir gradualmente en la menopausia.

Entre las mujeres sometidas a biopsia endometrial o histerectomía, la prevalencia de pólipos endometriales oscila entre $10 \%$ y $24 \%$ (5). La mayoría de ellos son benignos, pudiendo ser asintomáticos y por ende no infrecuentemente un hallazgo de la ultrasonografía pélvica o del estudio histológico en piezas quirúrgicas de cirugías indicadas por otra causa.

Al igual que el resto del endometrio, los pólipos poseen receptores de estrógenos y progesterona (6), y su génesis se ha asociado a una mayor exposición estrogénica. Por esto, condiciones donde exista un exceso de estímulo estrogénico se consideran factores de riesgo para su desarrollo. Dentro de ellas, el uso prolongado de tamoxifeno (por su efecto agonista del receptor de estrógenos en el endometrio) $(7,8)$, la obesidad $(9,10)$, la resistencia a la insulina (11) y la terapia de reemplazo hormonal con dosis altas de estrógenos o en asociación a progestinas con bajo efecto anti-androgénico $(12,13)$. Pese a todo, para estos factores avalados por la teoría, la evidencia disponible aún es insuficiente como para confirmar su rol en la génesis de los pólipos endometriales.

En nuestro centro, Merino y cols (4), demostraron en un estudio de incidencia de patología benigna, que la incidencia de pólipo endometrial no es baja en pacientes sometidas a histerectomía. En 5.683 histerectomías analizadas para el período 1991-2005, la incidencia de pólipo endometrial alcanzó el 7,4\% y sólo en el 2,4\% de ellos se demostró la presencia de cáncer. Esta serie confirmó que el pólipo endometrial es frecuente en mujeres que requieren tratamiento de histerectomía por patología ginecológica y que la mayoría de las veces corresponde a una entidad benigna. En un análisis ulterior, de la casuística de nuestro centro para dicho período, se revisaron los casos de las 409 pacientes sometidas a cirugía por sospecha de pólipo endometrial. Ninguno de los casos correspondió a pacientes adolescentes, todas ellas eran mujeres mayores de 30 años (rango: 32-84 años).

El objetivo de esta comunicación es presentar el primer caso registrado en nuestro centro de sangrado menstrual anormal en una adolescente debido a pólipo endometrial.

\section{Caso clínico}

Adolescente de 13 años, eutrófica (IMC: 19,9 $\mathrm{kg} / \mathrm{m} 2$ ), con antecedente de resistencia a la insulina en tratamiento con dieta, que consulta por sangrado genital anormal. Menarquia 10 meses previo a la consulta de características normales. Refiere menstruaciones regulares hasta los últimos 2 meses, cuando inicia episodios de metrorragia. Evaluada en otro centro se inicia manejo con dosis elevadas de anticonceptivos orales. Durante esa consulta se toma biopsia del material encontrado en la vagina que revela "endometrio menstrual". Pese a lograrse una reducción en el volumen de sangrado, la paciente persiste con metrorragia y ciclos irregulares, objetivándose un descenso del hematocrito hasta $34,5 \%$.

Al ingreso a nuestra unidad, la adolescente se encontraba en buenas condiciones generales, sin signos clínicos de anemia, ni de endocrinopatía. Las pruebas de coagulación: tiempo de protrombina, TTPK, tiempo de sangría, estudio de agregación plaquetaria, recuento de plaquetas y estudio de enfermedad de Von Willebrand, eran todas en rangos normales. Frente a ello se indicó progesterona para control de sus ciclos, atribuyendo los episodios de sangrado a la inmadurez fisiológica del eje hipotálamo-hipófisis-gonadal.

Se mantiene estable por 6 meses, y vuelve a consultar por nuevo episodio de metrorragia. En esta ocasión, la ultrasonografía pelviana demostró un engrosamiento del endometrio de $32 \mathrm{~mm}$. Hemoglobina de $10 \mathrm{mg} / \mathrm{dL}$. Nuevamente se manejó con altas dosis de anticonceptivos orales para controlar el sangrado y hierro oral para tratamiento de la anemia. Pese a ese manejo médico, la paciente persistió con goteo y el control con ultrasonografía pelviana vía 
abdominal demostró la persistencia de un endometrio engrosado e irregular. En opinión del clínico que evaluó las imágenes, dicho engrosamiento impresionaba causado por la presencia de una lesión focal, probablemente un pólipo (Figura 1).

Frente a esa hipótesis, se indicó estudio con histeroscopia, previo consentimiento informado de los padres. Previa preparación la noche anterior del cuello uterino con 200 ug de misoprostol administrados por vía oral, se procedió a la dilatación del cuello uterino con bujías de Hegar y posteriormente a la realización de una histeroscopia sin incidentes. El hallazgo fue la presencia de dos pólipos endometriales, los cuales fueron resecados vía histeroscópica, completándose el procedimiento con un legrado. El estudio anátomopatológico confirmó el diagnóstico de pólipos endometriales y un endometrio bajo el efecto de progestágenos (Figuras 2, 3 y 4). La ultrasonografía pelviana de control mostró un endometrio normal. Desde entonces, la paciente ha evolucionado favorablemente, reanudando un ritmo menstrual cíclico, con flujo normal en cantidad y duración y con resolución de la anemia.

\section{DISCUSIÓN}

Uno de los motivos de consulta más frecuentes en pacientes adolescentes es el sangrado genital anormal debido a trastornos menstruales. De hecho no es raro, que las adolescentes, en sus primeros años posmenarquia, presenten ciclos irregulares, asociados a sangrado abundante, los cuales motiven una consulta y que incluso determinen la aparición de anemia secundaria (14).

En este escenario, la primera causa a considerar es la inmadurez del eje hipotálamo-hipófisisgonadal. El origen de este trastorno no es aún del todo claro. Sin embargo, sí es importante reconocerla como la causa más frecuente de sangrado asociado a trastornos menstruales en la adolescencia y que su diagnóstico debe ser de descarte, particularmente si la magnitud del sangrado es importante (14).

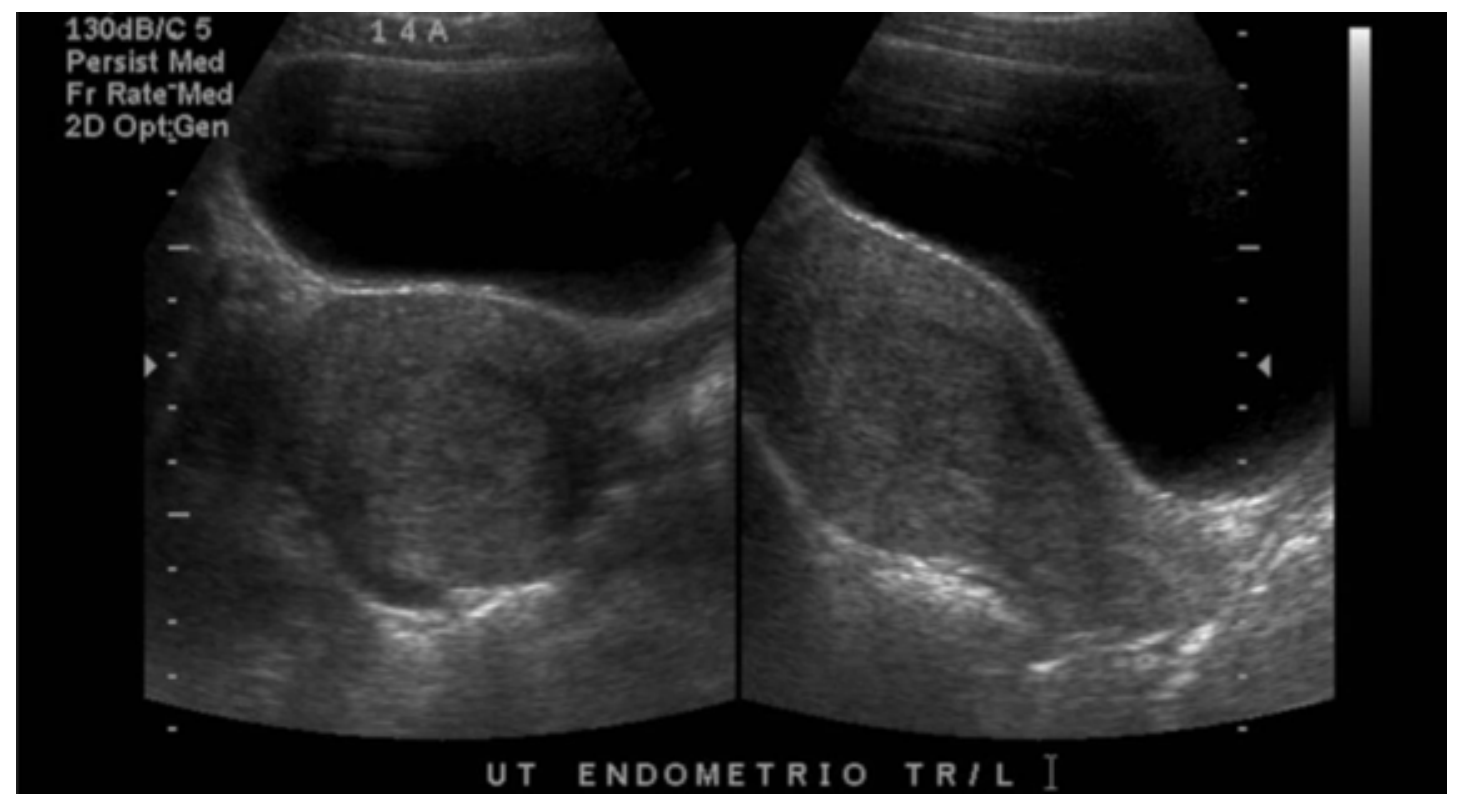

Figura 1. Imágenes de ultrasonografía pelviana transabdominal. Se muestra los cortes transversal y longitudinal del cuerpo uterino donde se evidencia un endometrio engrosado. 


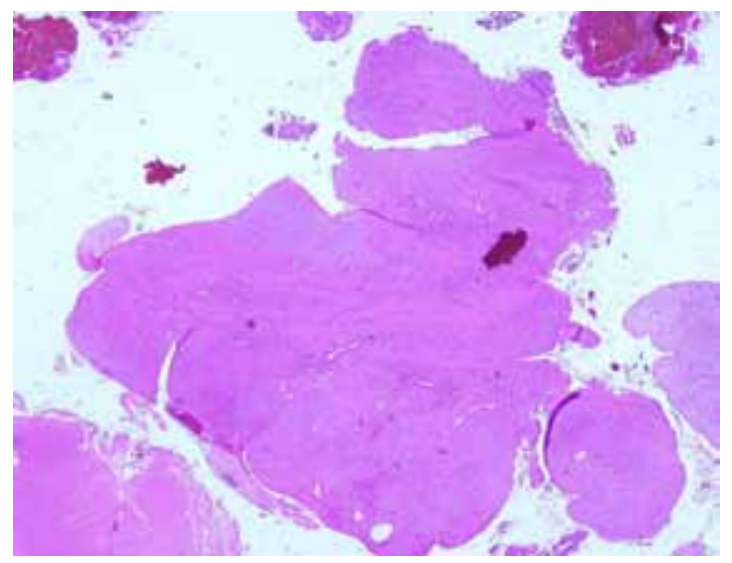

Figura 2. Microfotografía mostrando pólipo endometrial (H-E, 20X). Se aprecia una formación polipoídea con componente glandular marcadamente atrófico, asociado a estroma exuberante, marcadamente decidualizado. La disociación entre el estroma y el endometrio ("endometrio disociado") es característica del efecto inducido por progestágenos.

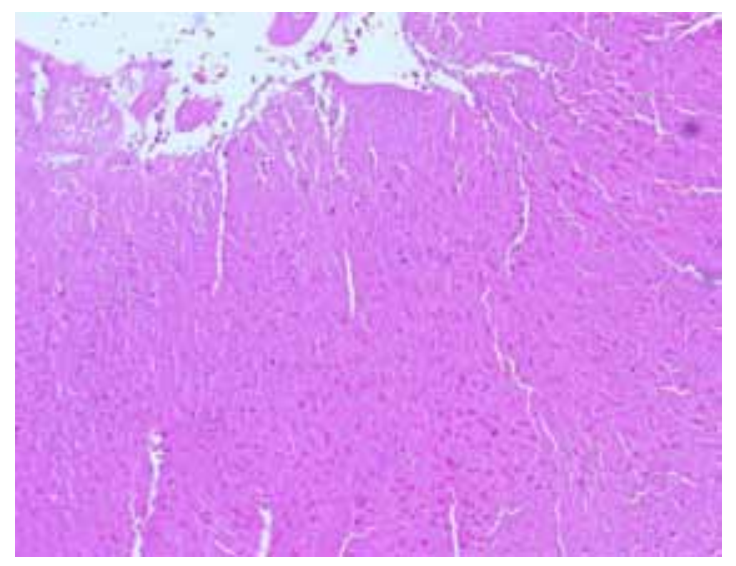

Figura 3. Microfotografía con magnificación de un área del pólipo endometrial (H-E, 100X). En ella se aprecia el endometrio bajo efecto de progestágenos. El estroma del pólipo muestra marcada decidualización y signos de involución.

Las causas de sangrado anormal en mujeres adolescentes son varias. Frente a la presencia de una paciente que consulta por este motivo es importante considerar siempre la historia clínica, el registro de sus ciclos menstruales previos, el momento de la menarquia, el examen físico, los exámenes

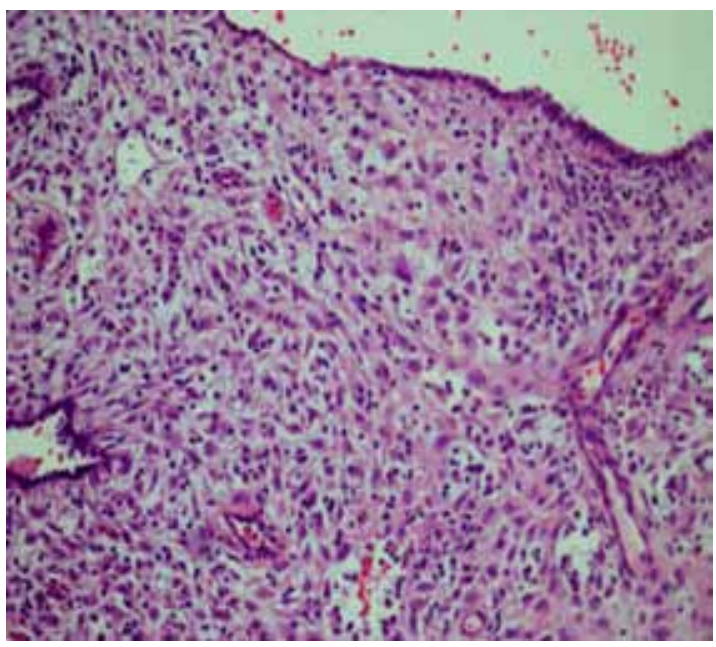

Figura 4. Microfotografía donde se muestra el endometrio bajo el efecto de progestágenos y la marcada decidualización y signos de involución en el estroma del pólipo (H-E, 200X).

de laboratorio y finalmente el estudio de imágenes para completar el estudio.

En pacientes con historia de ciclos menstruales regulares y de cuantía normal, las cuales de un momento a otro presentan un sangrado anormal, es importante tener en consideración causas menos comunes que pudiesen explicar dicho trastorno. En este contexto debemos descartar siempre la posibilidad de un embarazo, la manifestación de trastornos de la coagulación y por último alteraciones anatómicas uterinas que pudiesen explicar un cambio en el patrón normal del ciclo menstrual.

En el caso de las alteraciones anatómicas uterinas, siempre se debiera sospechar la presencia de miomas o de un pólipo endometrial, como es el caso de nuestra paciente. Debe recordarse que pese a su baja incidencia, al igual que en las mujeres adultas, ambas patologías también se puede presentar en la adolescencia.

A la fecha existen pocos casos reportados de pólipo endometrial en adolescentes. Como lo mencionamos en la introducción, corresponde a una patología cuya prevalencia aumenta cercano a la perimenopausia y que infrecuentemente se presenta en la adolescencia. Es por ello que muchas veces los clínicos no consideran en el diagnóstico diferencial a esta condición.

En adolescentes con trastorno menstrual, el estudio debiese incluir una ultrasonografía pelviana ya sea abdominal o transvaginal, a fin de evaluar 
la normalidad anatómica del útero y los anexos, así como también las características del endometrio, en particular su grosor. La elección de una vía u otra dependerá si la paciente ha iniciado o no actividad sexual. Lo habitual a esta edad es encontrar un útero de dimensiones normales para la edad, con ovarios funcionales, no infrecuentemente conteniendo múltiples folículos pequeños, y un endometrio de dimensiones y características similares al observado en pacientes adultas dependiendo de la etapa del ciclo, pero donde muchas veces predomina el aspecto proliferativo, propio del estímulo estrogénico de ciclos anovulatorios. En este contexto, la gran mayoría de las adolescentes no presentará anormalidad, razón por la cual aún no existe consenso si la ultrasonografía es costo efectiva y si debe ser incluida en el estudio inicial $(15,16)$. Sin embargo, frente a la falta de respuesta al tratamiento médico se debe sospechar la presencia de una lesión focal. Es ahí donde el estudio ultrasonográfico constituye la mejor herramienta alternativa de evaluación $(14,17)$.

Frente a la sospecha de una lesión focal (pólipo o mioma submucoso) como causa del sangrado anormal, la confirmación diagnóstica se debe realizar por medio de la histeroscopia. En pacientes menores de edad, es importante recordar que además de obtener la aceptación del examen y procedimiento por parte de la paciente, se debe obtener el consentimiento informado de los padres. En aquellas adolescentes que no han iniciado actividad sexual, tanto el examen genital como la histeroscopia debe realizarse bajo anestesia.

Por consenso, todos los pólipos sintomáticos deben ser resecados, independiente de la edad que tenga la paciente (13). El ideal es realizar la resección por vía histeroscópica del pólipo y enviar el material obtenido a estudio histológico. En adolescentes debiese evitarse el legrado vigoroso del endometrio circundante a la lesión a fin de evitar cicatrices que puedan comprometer la fertilidad futura.

El estudio histológico no solo se justifica con el fin de confirmar el pólipo endometrial sino además la posibilidad de malignidad. En mujeres adultas, la mayor incidencia de malignidad se ha asociado al uso prolongado de tamoxifeno y a pólipos de gran tamaño (en general mayor a $15 \mathrm{~mm}$ de diámetro) $(18,19,20)$. La ocurrencia de cáncer asociado a pólipo en la adolescencia pareciera ser excepcional considerando la baja frecuencia de ambas entidades en este grupo etario. De hecho, hasta la fecha sólo existe un caso reportado de cáncer endometrial asociado a pólipo endometrial hiperplástico en una mujer con síndrome de Turner, con menarquia espontánea y reglas irregulares por 9 años, a quien se le diagnosticó el pólipo asociado a cáncer a los 21 años (21).

Pese a su baja incidencia en la adolescencia, los pólipos endometriales debiesen ser considerados en el diagnóstico diferencial en adolescentes que consultan por trastornos menstruales, particularmente si la anormalidad persiste posterior al tratamiento hormonal. En dicho escenario, el uso del ultrasonido pélvico debiese ser de gran utilidad en la búsqueda de una lesión focal como causa del problema.

Según las cifras de la Organización para la Cooperación y el Desarrollo Económico (OCDE) del año 2011 , el 27,1\% de las niñas entre 5 y 17 años presentan sobrepeso y obesidad en Chile, alcanzando una cifra global de $21,4 \%$ para los países pertenecientes a dicha organización (22). Refrendando esta estadística, el registro de la prueba SIMCE de educación física mostró que el $40 \%$ de los adolescentes de $8^{\circ}$ básico está excedido de peso. Considerando que la obesidad aumenta el riesgo de anovulación y la exposición a exceso de estrógenos, y que ambas condiciones aumentarían el riesgo de desarrollar pólipos endometriales, es esperable que esta condición aumente su incidencia en población adolescente. Particularmente en esta paciente, si bien tenía IMC normal al momento del diagnóstico, ella presentaba una alteración metabólica que forma parte de la fisiopatología de la obesidad, esto es resistencia a la insulina, alteración que también se ha relacionado con la génesis de pólipos endometriales. Considerando el cambio del perfil epidemiológico de la población chilena, la mayor prevalencia de alteraciones metabólicas que podrían condicionar una mayor incidencia de pólipos, creemos importante el tener presente al pólipo endometrial como causa, aunque infrecuente, de trastornos menstruales en adolescentes.

\section{REFERENCIAS}

1. Appelbaum $\mathrm{H}$, Acharya SS. Heavy menstrual bleeding in adolescents. Minerva Gynecologic 2001;63:547-61.

2. Chung PW, Chan SS, Yiu KW, Lao TT, Chung TK. Menstrual disorders in a paediatric and adolescent gynaecology clinic: patient presentations and longitudinal outcomes. Hong Kong Med J 2011;17:391-7.

3. Bevan JA, Maloney KW, Hillery CA, Gill JC, Montgomery RR, Scott JP. Bleeding disorders: a common cause of menorrhagia in adolescents. J Pediatrics 2001;138:856-61.

4. Merino P, Doren A, Saez N, Valenzuela P, Etchegaray A, Gejman R, Cuello M. Distribución de la patología quirúrgica de cuerpo uterino en mujeres chilenas sometidas a histerectomía en un hospital clínico universitario. Rev Chil Obstet Ginecol 2009;74:15-29. 
5. Catalán A, Corvalán J, Pantoja V, García K, Godoy M. Pólipos endometriales: manejo con histeroscopia quirúrgica y correlación histológica. Rev Chil Obstet Ginecol 2007;72:116-9.

6. Gul A, Ugur M, Iskender C, Zulfikaroglu E, Ozaksit G. Immunohistochemical expression of estrogen and progesterone receptors in endometrial polyps and its relationship to clinical parameters. Arch GynecolObstet 2010;281:479-83.

7. Cohen I. Endometrial pathologies associated with postmenopausal tamoxifen treatment. Gynecol Oncol 2004;94:256-66.

8. 8-Runowicz CD, Costantino JP, Wickerham DL, Cecchini RS, Cronin WM, Ford LG, Vogel VG, Wolmark N. Gynecologic conditions in participants in the NSABP breast cancer prevention study of tamoxifen and raloxifene (STAR). Am J Obstet Gynecol 2011;205:535. e1-5

9. Oguz S, Sargin A, Kelekci S, Kelekci S, Aytan H, Tapisiz OL, et als. The role of hormone replacement therapy in endometrial polyp formation. Maturitas 2005;50:231-6.

10. Onalan R, Onalan G, Tonguc E, Ozdener T, Dogan $M$, et als. Body mass index is an independent risk factor for the development of endometrial polyps in patients undergoing in vitro fertilization. Fertil Steril, 2009;91:1056-60.

11. Kacalska-Janssen $O$, Rajtar-Ciosek A, Zmaczyński A, Wyroba J, Milewicz T, Krzyczkowska-Sendrakowska $\mathrm{M}$, Krzysiek J. Markers of insulin resistance in perimenopausal women with endometrial pathology. Ginekol Pol 2013;84:922-9.

12. Maia H Jr, Maltez A, Studard E,Athayde C, Couthino EM. Effect of previous hormone replacement therapy on endometrial polyps during menopause. Gynecol Endocrinol 2004;18:299-304.
13. Wolfman W, Leyland N, Heywood M, Singh SS, Rittenberg DA, Soucy $R$, et als. Asymptomatic endometrial thickening. J Obstet Gynaecol Can 2010;32:990-9.

14. Hayden Gray S, Emans J. Chapter 10: Abnormal vaginal bleeding in the adolescent. Emans \& Laufer, Goldstein's Pediatric and Adolescent Gynecology, 6th edition.Emans J, Laufer M, Eds. Lippincott Williams \& Wilkins, 2012; pp159-167.

15. Boswell HB. The adolescent with menorrhagia: why, who and how to evaluate for a bleeding disorder. J Pediatr Adolesc Gynecol 2011;24:228-30.

16. Davis VJ, Dizon CD, Minuk CF. Rare cause of vaginal bleeding in early puberty. J Pediatr Adolesc Gynecol 2005;18:113-5.

17. Garel L, Dubois J, Grignon A, Filiatrault D, Van Vliet G. US of the pediatric female pelvis: a clinical perspective. Radiographics 2001;21:1393-1407.

18. Baiocchi G, Manci N, Pazzaglia M, et al. Malignancy in endometrial polyps: a 12-year experience. Am J Obstet Gynecol 2009;201:462.e1.

19. Ferrazzi E, Zupi E, Leone FP, Savelli L, Omodei U, Moscarini $M$, et als. How often are endometrial polyps malignant in asymptomatic postmenopausal women? A multicenter study. Am J Obstet Gynecol 2009;200:235.e1-6

20. Ben-Arie A, Goldchmit C, Laviv Y, Levy R, Caspi $\mathrm{B}$, Huszar $\mathrm{M}$, et als. The malignant potential of endometrial polyps. Eur J Obstet Gynecol Reprod Biol 2004;115:206-210.

21. Kocova M, Basheska N, Papazovska A, Jankova R, Toncheva D, Popovska S. Girls with Turner's syndrome with spontaneous menarche have an increased risk of endometrial carcinoma: a case report and review from the literature. Gynecol Oncol 2005;96:840-5.

22. Sassi F, Devaux M. Obesity Update 2012. OECD Health Division. Disponible en: http://www.oecd.org/ health/49716427.pdf. Acceso: 10 de mayo 2014 